\title{
Recién nacido hijo de madre diabética
}

\author{
Mariano García $\mathrm{C}^{1}$; Gabriela Kunstmann $\mathrm{Z}^{2}{ }^{2}$; Nelson Vargas $\mathrm{C}^{2}$; \\ Gloria López S.
}

\author{
Infants born to diabetic mothers
}

\begin{abstract}
Some features as well as pathological findings in 63 infants born to diabetic mothers \{|DM\} under control in a diabetes unit of a general hospital at metropolitan Santiago. Chile, from 1985 through 1988 are presented. Out of 63 newborns, 22 came from mothers with pregestational diabetes mellitus (PGDM) and 41 were born to mothers with gestational diabetes meilitus (GDM). Significant differences were detected among PGDM and GDM groups of newborns in the following features: mean gestational age $\{G E\}$ prematury $154.5 \%$ vs. $26.8 \% 37$ weeks GE or less\}, jaundice $(40.9 \%$ vs. $17.1 \%)$ and congenital malformations $(18.2 \%$ vs. $0 \%)$. No significant differences were found in fetal distress, hypoglicemia, respiratory distress syndrome and birth trauma although as a whole these last were more frequently recorded among infant born 10 insulin dependent diabetic mothers. It is concluded that in spite of an appropriate control, morbiaity remains higher in IOM, particulary in IDM PGDM.

(Key words: infant, newborn, diabetic mother, diabetes mellitus.)
\end{abstract}

El recién nacido (RN) de madre diabética (HMD) presenta características morfológicas, fj. siológicas y patológicas especiales ${ }^{1}$, pues al desarrollarse en un medio ambiente distinto al normal, se favoreceria en ellos un anabolismo exa. gerado, que seria atribuible a la coexistencia de hiperglicemia e hiperinsulinismo ${ }^{2}$.

El riesgo de estos niños tiene relación con la gravedad de la diabetes materna $\mathrm{y}$ su relación con el mornento en que ésta aparece con respecto a la gestación: antes del embarazo (DPG) o en su transcurso (DG). Estas variables deben ser consideradas en el manejo clínico y epidemiológico ${ }^{3}$.

Con el advenimiento de mejores cuidados obstétricos y neonatales y el desarrollo de programas médicos para control, seguimiento $y$ tratamiento de estas gestantes, se ha logrado reducir la morbimortalidad del HMD ${ }^{4,5}$.

El propósito de este trabajo fue describir las complicaciones de HMD controladas en un consultorio de diabetes, asociado a un hospital

1. Médico en especialización en Pediatría, Departamento de Pediatría y Cirugía Infantil Occidente, Facultad de Medicina, Universidad de Chile.

2. Departamento de Pediatría y Cirugía Infantil, Facultad de Medicina, Universidad de Chile.

3. Unidad de Diabetes, Servicio de Medicina Interna, Hospital San Juan de Dios. general metropolitano de Santiago, a lo largo de cuatro años, $y$ efectuar una comparación entre HMD pregestacional (HMD PG) y HMD gestacional (HMD G), en busca de algunas diferencias de interés clínico entre ambos grupos.

\section{Material y Método}

La fuente de datos consistió en 63 historias clínicas de diabéticas embarazadas, controladas en el Departamento de Diabetes del hospital San Juan de Dios de Santiago, Chile, $y$ en las de sus hijos recién nacidos. El control de las madres se hizo de acuerdo a un esquema de la misma unidad. Los njĩos necieron en la maternidad del hospital entre el 1 de enero de 1985 y el 31 de dísjembre de 1988 .

La edad gestacional (EG) fue determinada por ed método de Parkin, en el momento del nacimiento. Se consideraron $R N$ pretérmino los nifios nacidos a las 37 semanas de EG o antes. Se consideró RN de peso adecuado para la EG a los niños cuyos pesos al nacer estaban comprendidos entre los percentiles 10 y 90 en las curvas de Lubchenco. A la vez, se calificó como macrosomia la ubicación sobre el percentil 90 , en las migmas curvas. Se definió como sufrimiento fetal a la presencia de uno o más de los siguientes parámetros: desaceleraciones de la frecuencia cardíaca ("Dips" tipo II); bradicardia fetal; meconio en el líquido amniótico. Se llamó trauma obstétrico a todas las lesiones del RN rejacionadas con el trabajo de parto, manipulaciones 0 instrumentación obstétrica. Se denominó hipoglicemia a toda glicemia inferior a $40 \mathrm{mg} / \mathrm{dl}$ en RN de término e inferior o igual a $30 \mathrm{mg} / \mathrm{dl}$ en $\mathrm{RN}$ 
pretérmino, en las 6 primeras horas de vida. Llamamos ictericia a la concentración sérica de bilirrubina de $12 \mathrm{mg} / \mathrm{dl}$ en el RN de tírmino y sobre $14 \mathrm{mg} / \mathrm{d}$ ] en RN pretérmino, en cuakquier momento de su evolución. Se detonió trastorno respiratorio a la presencia de sig nos clinicos (retracción costal, aleteo nasal y quejido) sumados a necesidad de aporte adicional de oxígeno para mantener una presión parcial arterial adecuada del gas. Se delinió como asfixia neonatal la calificación de Apgar (al minuto) menor o igual a 6 puntos 6 , sumada a acidosis metabólica con déticit de base mayor a 10. Se consideraron malformaciones mavores las capaces do comprometer la vida del paciente o la calidad de la misma, y menores a las restantes.

El análisis estadístico se hizo, en las variables cuantitativas, a través del cálculo de " $t$ " de Student; en las cualitativas se calculó Chi cuadrado y se determinó el valor de $Z$, para las variaciones entre porcontajes. El nivel de significación estadística para rechazar la hipótesis nula se estableció en alfz $=0,5$ unilateral.

\section{Resultados}

E1 sexo de $58,7 \%$ de los RN era femenino. La distribución de la diabetes materna fue clasificada, según White ${ }^{7}$, siendo más frecuente el grupo A, que engloba las diabetes gestacionales, $y$ es una forma latente en que las exigencias del embarazo revelarán una prueba positiva de tolerancia. Al anterior siguen los grupos B, $C, D, F$ y $R$, que son diabetes pregestacionales.

En lo referente a edad y paridad, entre las
Tabla 1

Edad y pariơad de las madres con diabetes pre y postgestacional

\begin{tabular}{|c|c|c|c|c|}
\hline & \multicolumn{2}{|c|}{ PG } & \multicolumn{2}{|c|}{$G$} \\
\hline & $\mathrm{n}$ & $\%$ & n & $\%$ \\
\hline \multicolumn{5}{|l|}{ Edad materna } \\
\hline $\begin{array}{l}- \text { de } 30 \\
30 \text { o más }\end{array}$ & $\begin{array}{r}(14) \\
(8)\end{array}$ & $\begin{array}{l}64 \\
36\end{array}$ & $\begin{array}{l}(10) \\
(31)\end{array}$ & $\begin{array}{l}24 \\
76\end{array}$ \\
\hline \multicolumn{5}{|l|}{ Paridad } \\
\hline $\begin{array}{l}- \text { de } 3 \\
3 \text { o más }\end{array}$ & $\begin{array}{r}(14) \\
(8)\end{array}$ & $\begin{array}{l}64 \\
36\end{array}$ & $\begin{array}{l}(13) \\
(2 B\end{array}$ & $\begin{array}{l}32 \\
68\end{array}$ \\
\hline
\end{tabular}

PG: diabetes pregestacional $\quad$ G: diabetes gestacional

madres DPG había 14 (64\%) con menos de 30 años, y $14(64 \%)$ con menos de tres hijos; en el grupo DG, 31 madres $(76 \%)$ tenían más de 30 años y $28(68 \%)$ tres o más hijos (tabla 1 ).

No hubo diferencias significativas entre ambos grupos de $\mathrm{RN}$, en el promedio de peso al nacer y la frecuencia de macrosomia (tabla 2), pero e1 tamaño corporal fue muy superior al que se observa en los hijos de la población ${ }^{8}$.

La hipertensión arterial y la preclampsia fueron las entidades nosológicas asociadas más frecuentes en las madres, en especial la primera de ellas en el grupo de DPG (tabla 3 ).

Tabla 2

Caracter ísticas somáticas de los hijos de madres diabéticas

\begin{tabular}{lccc}
\hline & HMD PG & HMD G & Total \\
\hline Peso (g) & $3.183 \pm 675$ & $3.427 \pm 666$ & \\
Macrosomía & $(6) \quad 27,3 \%$ & $(13) 31,7 \%$ & (19) $30,0 \%$ \\
Adecuación de peso & $(16) 72,7 \%$ & $(27) 65,3 \%$ & (43) $68,2 \%$ \\
\hline
\end{tabular}

HMD : hijo de nuadres diabéticas. PG: diabetes pregestacional. G: diabetes gestacional.

Tabk 3

Afecciones de las madres diabéticas durante el embarazo

\begin{tabular}{|c|c|c|c|c|c|c|}
\hline & \multicolumn{2}{|c|}{ PG } & \multicolumn{2}{|c|}{ G } & \multicolumn{2}{|c|}{ Total } \\
\hline & $\mathbf{n}$ & * & $\mathbf{n}$ & 4 & $n$ & $\%$ \\
\hline Hipertensión & (13) & 59,1 & (15) & 36,6 & (28) & 44,4 \\
\hline Preeclampsia & (8) & 36,4 & (12) & 29,3 & (20) & 31,7 \\
\hline Infección urinaria & (10) & 45,4 & (9) & 21,9 & (19) & 30,1 \\
\hline Polihidramnios & (6) & 27,3 & (4) & 9,7 & (10) & 15,9 \\
\hline Rotura prematura de membranas & (7) & 31,8 & (s) & 12,2 & (12) & 19,0 \\
\hline
\end{tabular}

PG: dabetes pregestacional. G: diabetes gestacional. 
Se observó menor EG y mayor incidencia de ictericia, malformaciones congénitas y prematuridad en el grupo HMD PG, con respecto al HMD G; diferencias que fueron significativas. Doce $(54,5 \%)$ HMD PG nacieron en edades gestacionales de 37 semanas o menos, cifra significativamente mayor a los 11 (26,8\%) del grupo de HMD G. Hubo, a la vez, diferencia significativa en la ictericia $(40,9 \%$ versus $17,1 \%)$, respectivamente. El trastorno respiratorio fue más frecuente en el grupo de hijos de madres con diabetes pregestacionales, pero sin alcanzar significación. El parto por cesárea y el sufrimiento fetal fueron más frecuentes en el grupo de madres diabéticas preges. tacionales, pero las diferencias no son significativas.

Entre los HMD PG, en $4(18,2 \%)$ se constataron malformaciones congénitas, dos de éstas fueron mayores: un $\mathrm{RN}$ con transposición de los grandes vasos y otro con miocardiopatía hipertrófica asimétrica. Las dos malfomaciones restantes fueron menores (sindactilia en un caso $y$ quiste branquial en otro). Entre los HMD G no se detectaron malformaciones. En las frecuencias de hipoglicemia, asfixia neonatal y trau. matismo de parto no se registraron diferencias significativas, a pesar de la tendencia que se exhibe en la tabla 4. En ambos grupos hubo un caso de muerte perinatal. En el grupo HMD PG $(4,5 \%)$ ésta fue debida a septicemia, en el segundo dia de vida, encontrándose en la anatomía patológica focos hemorrágicos múltiples suprarrenales y subaracnoideos. En el grupo HMD G $(2,4 \%)$ hubo un mortinato con signos de infec. ción ovular. La mortalidad perinatal total fue 3,2\%. En el año 1988 ésta alcanzó a 1,2\% de la población general del área.
Hubo necesidad de hospitalización en 16 casos de HMD PG $(72,7 \%)$ y 11 HMD G $(26,8 \%)$; el promedio de dias de estada en el hospital fue de 7.6 y 9,6 , respectivamente.

\section{Discusión}

En las últimas decadas, debido fundamentalmente al buen manejo de la mujer gestante diabética y del hijo recién nacido, se ha logrado una reducción significativa en la mortalidad perina. tal, desde 60\% antes del empleo de insulina, hasta proporciones actuales de 2 a $4 \%{ }^{\circ}$. El mecanismo preciso de muerte fetal es desconocido, aunque algunos autores lo atribuyen a hipoxia crónica intrauterina ${ }^{10}$ producida por me. nor oxigenación de la hemoglobina y reduccion del flujo sanguíneo placentario (esta última asociada fundamentalmente a preeclampsia y cetoaçidosis maternas). Entre las causas posibles de muerte neonatal figuran las malformaciones congénitas y las enfermedades respiratorias ${ }^{11}$. En nuestro estudio la letalidad total fue similar eritre ambos grupos, siendo las infecciones la única causa de muerte perinatal. Algunos trabajos describen complicaciones infecciosas en 17\% de los casos, pero no la consideran entre las causas de muerte ${ }^{12}$.

$\mathrm{La}$ frecuencia de malformaciones congénitas mayores en los HMD PG fue semejante a la descrita en publicaciones extranjeras ${ }^{13,14}$; no obstante, ellas no fueron detectadas entre los HMD G, a quienes se atribuye frecuencias de 3 a $4,5 \%{ }^{4}$. Se ha sugerido, como causa de malformaciones, el control metabólico inadecuado en las siete primeras semanas de gestación, junto con cierta susceptibilidad genética ${ }^{15,16}$, En

Tabla 4

Trastornos neonatales en tijos de madres djabéricas con diabetes pregestacional y gestacional

\begin{tabular}{|c|c|c|c|c|c|c|c|}
\hline & \multicolumn{2}{|c|}{ HMD PG } & \multicolumn{2}{|c|}{ HMD G } & \multicolumn{3}{|c|}{ Total } \\
\hline & $\mathrm{n}$ & $\%$ & $n$ & $\%$ & n & $\%$ & \\
\hline Prematuridad & (12) & 54,5 & (II) & 26,8 & (23) & 36,5 & $S^{*}$ \\
\hline Ictericia & (9) & 40,9 & (7) & 17,1 & (16) & 25,4 & $\mathrm{~S}^{*}$ \\
\hline Sufrimjento fetal & (4) & 18,2 & (2) & 4,9 & (6) & 9,5 & \\
\hline Malformación congénita & (4) & 18,2 & - & - & (4) & 6,3 & $\mathrm{~S}^{*}$ \\
\hline Sindrome de dificultad respiratoria & (4) & 18,2 & (2) & 4,9 & (6) & 9,5 & \\
\hline Hipoglicemia & (6) & 27,3 & (5) & 12,2 & (11) & 17,5 & \\
\hline Trauma obstétrico & (2) & 9,1 & (2) & 49 & (4) & 6,3 & \\
\hline Asfixin & (2) & 9,1 & (1) & 2,4 & (3) & 4,8 & \\
\hline
\end{tabular}

$\mathrm{S}^{*}$; significativo. HMD: hijo de madres diabéticas. PG: diabetes pregestacional. G: diabetes gestacional. 
nuestro medio, a diferencia de otros traba$\operatorname{jos}^{4,13}$, no detectamos malformaciones neurológicas.

Respecto a la relación del peso con la EG es evidente la existencia de una alta proporción de macrosomía, en coincidencia con otras casuísti$\operatorname{cas}^{17,18}$. Esto explicaría parcialniente el alto porcentaje de cesáreas de ambos grupos $(58,7 \%)$, hallazgo también atribuible al sufrimiento fetal $y$, quizás más importante, a las afecciones de estas madres. Este hecho también explica el elevado número de partos prematuros en ambos grupos, especialmente en los HMD PG, cuyas madres tuvieron más complicaciones.

En el trastorno respiratorio de estos pacientes influirian el porcentaje alto de prematuri$\operatorname{dad}^{6}$ y los niveles elevados de insulina en el feto, que interferirian en las síntesis de surfactante ${ }^{19}$, inhibiendo la maduración pulmonar inducida por los glucocorticoides. Un buen manejo de la gestante djabética debería ser capaz de equiparar la incidencia de esta complicación a la observada en hijos de madres sin diabetes ${ }^{6}$.

La incidencia de ictericia -similar a la observada por otros autores-sería atribuible fundamentalmente a la prematuridad ${ }^{4,20}$.

La hipoglicemia en'el recién nacido está influi. da por el control de la glicemia materna durante la segunda mitad del embarazo y la magnitud de ésta durante el parto. Glicemias maternas, durante el parto, sobre $90 \mathrm{mg} / \mathrm{dl}$, aumentan la frecuencia de hipoglicemia neonatal ${ }^{20}$. En nuestra casuistica encontramos alta incidencia de hipoglicemia tanto en hijos de madres con diabetes gestacionales como pregestacionales.

La asfixia neonatal fue importante en ambos grupos, $e$ igualmente ha sido relacionada con prematuridad e hiperglicemia durante el trabajo de parto. Se han descrito reducciones del flujo placentario hasta en $45 \%$ en esas condicio. nes ${ }^{6}$.

Los hijos de madres diabéticas deben ser considerados recién nacidos de alto riesgo, por su elevada morbimortalidad. En todo RN HMD debemos buscar enfermedades neonatales: malformaciones congénitas, hipoglicernia, hiperbilirrubinemia, entre otras. Los HMD PG presentan mayor frecuencia de morbilidad que los HMD G.

Un buen control metabólico de la diabetes, antes y durante la gestación, así como en el parto, disminuyen las complicaciones en los recién nacidos y mejoran su pronóstico. Para obtenerlo es conveniente desarrollar relaciones óptimas entre los departamentos de diabetes, obstetricia y pediatría.

\section{Resumen}

Se describe la morbilidad de hijos de madres diabéticas (HMD), en pacientes controladas en la unidad de diabetes del hospital San Juan de Dios, en Santiago, Chile, durante el periodo 1985-1988. De un total de 63 recién nacidos (RN) HMD, 22 nacieron de 20 madres con diabetes pregestacional (DPG) y 41 de 39 madres con diabetes gestacional (DG). Según el tipo de diabetes materna se detectaron diferencias sig. nificativas en los RN en las siguientes variables: edad gestacional, prematuridad, ictericia y malformaciones congénitas. Todas las complicaciones anteriores fueron más frecuentes en el HMD $P G$, destacando un porcentaje elevado de malformaciones $(18,2 \%)$. No se encontraron diferencias significativas en sufrimiento fetal, hipoglicemia, sindrome de dificultad respiratoria $y$ trauma de parto, aunque todos fueror más frecuentes en el grupo DPG. Se concluye que, a pesar de un buen control médico de la enfermedad de base, la morbilidad sigue siendo elevada en los $\mathrm{HMD}$, y mayor aún en $\mathrm{HMD} P \mathrm{PG}$.

(Palabras clave: recièn nacido, madre diabética, diabetes mellitus.)

\section{Agradecimientos}

A las doctoras Sibya Vives A. e Iva Camacho $N_{\text {, }}$, de la Unidad de Diabetes del Hospital San Juan de Dios.

\section{Referencias}

1. Martinez, P.: Gallo, A.; Schilling, E.: Reyes, E.; Gallegos, $R$ : Hijo de madre diabética. Rev Chil Pediatr $1981 ; 32: 469-476$.

2. Petersen, $I_{\text {ir }}$ Bojen-Haller, B.: Poulsen, H.: Blood sugar in newborn infants of diabetic mothers: Acra Endocrinol. 1954; 15: 33-52.

3. Valenzuele, M.A.; Argomedo, G.; Guajardo, H.; Peflo, E.; Castillo, R.: Experiencia clínica en diabetes y embarazo. Rev Med Chile 1989;117:508-515.

4. Pastor, X.: Jorba, J.M.: Cnuz, $O$. et al.: Recién nacido de madre diabética. Estudio clínico del periodo 1980-1985. Ann Esp Pediatr 1987; 27: $431-434$.

5. Tsang, R.: Ballord, J: Braun, C.: The infant of diabetic mothers today and tomorrow. Clin Obstet Gynecol 1981;24: 125-147. 
6. Mimouni, F, Miodounik, M.; Witsett, J.A.; Holroyde, J.C.: Siddiqui, T.A.; Tsang, R.C.: Respiratory distress syndrome in infants of diabetic mothers in the 1980: no direct adverse effect of maternal diabetes with modern management. Obstet Gynecol 1987;69: 191-5.

7. White, $P$ : : Pregrancy complicating diabetes. Am J. Med 1949; 7: 609-620.

8. Vargas, $N_{\text {. }}$ Thomas, E.; Rios, J. et al.: Peso de nacimiento. Hospital San Juar de Dios. 1984. Bol Hosp \$ J de Dios 1985; 32 : 379-386.

9. Lemons, J.A.: Varjos, P.: Deloneg, J.J.: Infant of diabetic mother. Review of 225 cases. Obstet Gynecol $1981 ; 106: 187-192$.

10. Madsen, M.: Fetal oxygenation in diabetic pregrancy. Dan Med Bull 1986; 33: 64-71.

11. Reller, M.D.; Tsang, R.C.; Meyer, R.A.; Braun. C.P.: Relationship of prospective diabetes control in pregnancy to neonatal cardjorespiratory function. J Pediatrics 1985; 106: 86-90.

12. Crespo, N.: Garcia, D.; Alamzan, M.; Mustelier, D.: Alvarez, $R$.: Complicaciones en hijos de madres diabéticas: estudio de 100 casos. Rev Cuba Pediatr 1986; 5B: $66+72$.

13. Simpion, J.L.: Elias, S.: Diabetes in pregnancy: Prospective study of anormalies in offspring of mothers with diabetes mellitus. Am J Obstet Gynecol 1983; 146: 283-291.
14. Mills, J.L; Knopp, R.H.; Simpson, J.L. et al.: Lack of relation of increased malfomation rates in infans of diabetic mothers to glycemic control during organogenesis. N Engl J Med 1988; 318 : $671-678$.

15. Lowy, $C_{. i}$ Beard, R.W.; Goldschmidr, J.: Congenital malformations in babies of diabetic mothers: Diabetic Med 1986; 3:458-462.

16. Rowe, B.R.; Rowbothom, C.J.; Bornett, A.M.: Preconception counselling, birth weight, and congenital abnormalities in established and gestational diabetic pregnancy: Diabetes Res (Scotland) $1987 ; 6: 33.39$.

17. Small, M.: Camerion, A.: Lunan, C.B.: Maciuish. A.C.: Macrosomia in pregnancy complicated by insulin-dependet mellitus: Diabetic Obstet Clinic $1987 ; 10: 594601$.

18. Lain, J.P.; Lovelace, D.R.: Clinical experience with 107 diabetes pregnancies. Am J, Obstet Gyrecol 1983; $147: 742-745$.

19. Engle, M.: Langan, S.M.: The effects of insulin and hyperglycemia on surfactant phospholipid biosynthesis in organotypic cuitures of type $\mathbf{U}$ pneumocytes. Biochem Biophys Acta 1983; 753 : 6-16.

20. Soler, N.G.; Soler, S.M.: Neonatal morbility among infants of diabetic mothers: Diabetes care 1978: $1: 340-349$. 